\title{
Biocompatibility of quantum dots (CdSe/ZnS ) in human amniotic membrane-derived mesenchymal stem cells in vitro
}

\author{
Gongping Wangä, Guangwei Zeng ${ }^{b \#}$, Caie Wangc, Huasheng Wang ${ }^{d}$, Bo Yange, \\ Fangxia Guan ${ }^{f}$, Dongpeng $\mathrm{Li}^{\mathrm{g}}$, Xiaoshan Feng ${ }^{\mathrm{h}}$
}

Background and Aim. Amniotic membrane-derived mesenchymal stem cells (hAM-dMSCs) are a potential source of mesenchymal stem cells which could be used to repair skin damage. The use of mesenchymal stem cells to repair skin damage requires safe, effective and biocompatible agents to evaluate the effectiveness of the result. Quantum dots (QDs) composed of CdSe/ZnS are semiconductor nanocrystals with broad excitation and narrow emission spectra, which have been considered as a new chemical and fluorescent substance for non-invasively labeling different cells in vitro and in vivo. This study investigated the cytotoxic effects of QDs on hAM-dMSCs at different times following labeling. Methods. Using $0.75,1.5$ and $3.0 \mu \mathrm{L}$ between quantum dots, labeled human amniotic mesenchymal stem cells were collected on days 1, 2 and 4 and observed morphological changes, performed an MTT cell growth assay and flow cytometry for mesenchymal stem cells molecular markers.

Results. Quantum dot concentration $0.75 \mu \mathrm{g} / \mathrm{mL}$ labeled under a fluorescence microscope, cell morphology was observed, The MTT assay showed cells in the proliferative phase. Flow cytometry expression CD29, CD31, CD34, CD44, CD90, CD105 and CD106.

Conclusions. Within a certain range of concentrations between quantum dots labeled human amniotic mesenchymal stem cells has good biocompatibility.

Key words: quantum dots, biocompatibility, human amniotic membrane, mesenchymal stem cells

Received: September 24, 2013; Accepted: August 13, 2014; Available online: October 2, 2014

http://dx.doi.org/10.5507/bp.2014.045

a Department of Oncological Surgery, the First Affiliated Hospital of Henan University of Science and Technology, LuoYang 471003, Henan Province, PR China

${ }^{b}$ Department of Plastic, Reconstructive and Burns Surgery, the First Affiliated Hospital of Henan University of Science and Technology, LuoYang 471003, Henan Province, PR China

'Department of Pharmacy, the First Affiliated Hospital of Henan University of Science and Technology, LuoYang 471003, Henan Province, PR China

${ }^{d}$ Department of Colorectal Surgery, People Hospital of Zhengzhou, Zhengzhou 450000, Henan Province, PR China 'Department of Neurosurgery, the First Affiliated Hospital of ZhengZhou University, ZhengZho 450001, Henan Province, PR China ${ }^{f}$ Henan Academy of Medical Sciences, ZhengZhou 450001, Henan Province, PR China

${ }^{9}$ Department of Emergency, the First Affiliated Hospital of Henan University of Science and Technology, LuoYang 471003, Henan Province, PR China

hDepartment of Oncological Surgery, the First Affiliated Hospital of Henan University of Science and Technology, LuoYang 471003, Henan Province, PR China

"The authors contributed equally to the work

Corresponding author: Xiaoshan Feng, e-mail: samfeng137@hotmail.com

\section{INTRODUCTION}

The skin undergoes a cycle of growth and degeneration to ensure the maintenance and homeostasis of adult skin. Stem cells residing in the skin participate in this cycle, but they also play an important role in repair of the epidermis after injury. Mesenchymal stem cells have been isolated from different tissues and have the ability to differentiate into epidermal-like cells ${ }^{1,2}$. Amniotic membrane-derived mesenchymal stem cells (hAM-dMSCs), with low immunogenicity and anti-inflammatory properties, have attracted attention as a novel source of stem cells, providing a potential source of mesenchymal stem cells to repair skin damage. Such repair requires the use of safe, effective and biocompatible agents to evaluate the effectiveness of the result. Different cell labeling reagents have been used, including biological dyes, radioactive reagents, and fluorescent protein antibodies among others ${ }^{3,4}$. Each of these labeling methods has its own disadvantages, such as low intensity, short labeling time and complicated procedures. Quantum Dots (QDs) are semiconductor nanocrystalloid particles which have a nano-scale character combined with a bright and stable emission spectrum, and can be used to label cells from different sources ${ }^{5-8}$.

We recently received nanoparticle quantum dots from Lanzhou University that are composed of $\mathrm{CdSe} / \mathrm{ZnS}$, enabling their detection with a fluorescence microscope ${ }^{9,10}$.

Nanotoxicology aims to evaluate the relationship between physical and chemical properties and toxic biological responses. Few data are available on the toxicity of QDs in human amniotic membrane-derived mesenchymal stem cells (hAM-dMSCs). We used biological approaches 
to evaluate the effects of QDs on hAM-dMSCs, including cell growth, cell viability and accumulation of QDs. The study investigated the effect of the cytotoxicity of QDs on hAM-dMSCs over different labeling durations. hAMdMSCs could be efficiently labeled at safe concentrations of QDs $(\leq 0.75 \mu \mathrm{g} / \mathrm{mL})$ without significantly affecting their viability ( $>80 \%$ by MTT assay with maintenance of normal morphology) or expression of specific surface antigens (CD29, CD44, CD90, CD105 analyzed by flow cytometry) from 1 to $4 \mathrm{~d}$.

\section{MATERIALS AND METHODS}

\section{Cell preparation}

This study was approved by the medical ethics board of the First Affiliated Hospital of Henan University of Science and Technology. Samples of amniotic membranes were obtained from full-term pregnant women during elective cesarean sections; each subject was fully informed of the procedure and consent was provided by each participant. Amniotic membranes were prepared according to the method previously described by Yang et al. ${ }^{11}$.

\section{Quantum dot (QD) labeling}

Quantum dots (QDs, Lanzhou University, PR China) were used in this study. Aliquots of $0.75,1.5$ and $3.0 \mu \mathrm{L}$ were removed from $1 \mathrm{~mL}$ of cytokine growth medium (CGM) which contains Dulbecco's modified eagle's medium-Low glucose (DMEM-LG; Gibco BRL, USA) and $10 \mathrm{ng} / \mathrm{mL}$ of basic fibroblast growth factor (bFGF; Sigma, USA), the corresponding volume of QDs suspension was added (final concentrations equivalent to $0.75,1.5$ and 3.0 $\mu \mathrm{L}$ QDs per $\mathrm{mL}$ ) to establish different concentrations of QDs. hAM-dMSCs cultured in CGM containing different concentrations of QDs were harvested at 1, 2 and $4 \mathrm{~d}$, and then divided into three parts: the first part was used to analyze the morphological and growth characteristics of the hAM-dMSCs; the second part was used for the 3-(4,5-dimethylthiazol-2-yl)-2,5- diphenyltetrazolium bromide (MTT) assay to assess the cytotoxicity of the QD suspension; the third part was used to detect the expression of surface antigens by hAM-dMSCs.

\section{Statistical analysis}

Data are expressed as the mean \pm standard error (SE), and one-way analysis of variance was used to compare the survival rates of hAM-dMSCs in the different groups. Differences were considered to be statistically significant when $P<0.05$.

\section{RESULTS}

\section{QD for imaging by fluorescence microscopy}

QDs emit a red color upon excitation with a single wavelength of light at $570 \mathrm{~nm}$ using a fluorescence microscope (DMIL; Leica, Germany). (Fig. 1 A). At the same wavelength, hAM-dMSCs stained with QDs $(1.5 \mu \mathrm{g} /$ $\mathrm{mL}$ ) when viewed under a fluorescence microscope at $1 \mathrm{~d}$ (Fig. $1 \mathrm{~B}, \mathrm{C}$ ).

\section{Growth characterization of hAM-dMSCs in primary culture}

hAM-dMSCs were prepared according to the method previously described by Yang et al. ${ }^{11}$, the cells retained their polygonal fibroblast-like morphological characteristics (Fig. 2) and maintained a strong mitotic competency to passage 10 .

\section{Surface antigens of hAM-dMSCs in primary culture \\ hAM-dMSCs cultured in CGM expressed CD29,} CD31, CD34, CD44, CD90, CD105 and CD106 (Fig. 3).

\section{Effects of QD-labeling on hAM-dMSCs}

Under different QD concentrations, QDs could be detected on the plasma membrance of hAM-dMSCs following different QD-labeling times. Inside cells, QDs still retained their spectral profile, while hAM-dMSCs was the excitation wavelength $570 \mathrm{~nm}$ under a fluorescence microscope following excitation (Fig. 1 B, C). Following 1 $\mathrm{d}$ of culture, the viability of cells labeled with QDs at various concentrations was evaluated. Cells labeled with QDs at concentrations $\leq 1.5 \mu \mathrm{g} / \mathrm{mL}$ were $>80 \%$ viable; at $2 \mathrm{~d}$, samples labeled with QDs at concentrations $\leq 0.75 \mu \mathrm{g} / \mathrm{mL}$ were > $80 \%$ viable; at $4 \mathrm{~d}$, samples labeled with QDs at concentrations $\leq 0.75 \mu \mathrm{g} / \mathrm{mL}$ were $>80 \%$ viable. Control samples that were not labeled with QDs were $>80 \%$ viable at 1, 2 and $4 \mathrm{~d}$ (Fig. 4). The polygonal fibroblast-like mor-
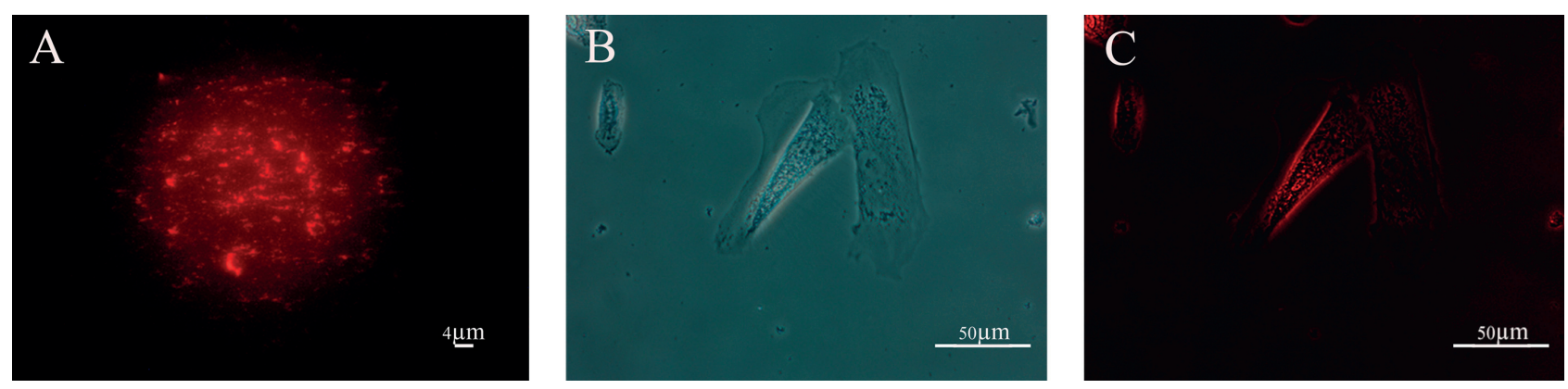

Fig. 1. Images of QDs and OD-labeled hAM-dMSCs. Using the single $570 \mathrm{~nm}$ wavelength of light of the fluorescence microscope, QDs (A) and QDs ( $1.5 \mu \mathrm{g} / \mathrm{mL}$, B before fluorescence microscope, C after fluorescence microscope ) taken by hAM-dMSCs at $1 \mathrm{~d}$. 

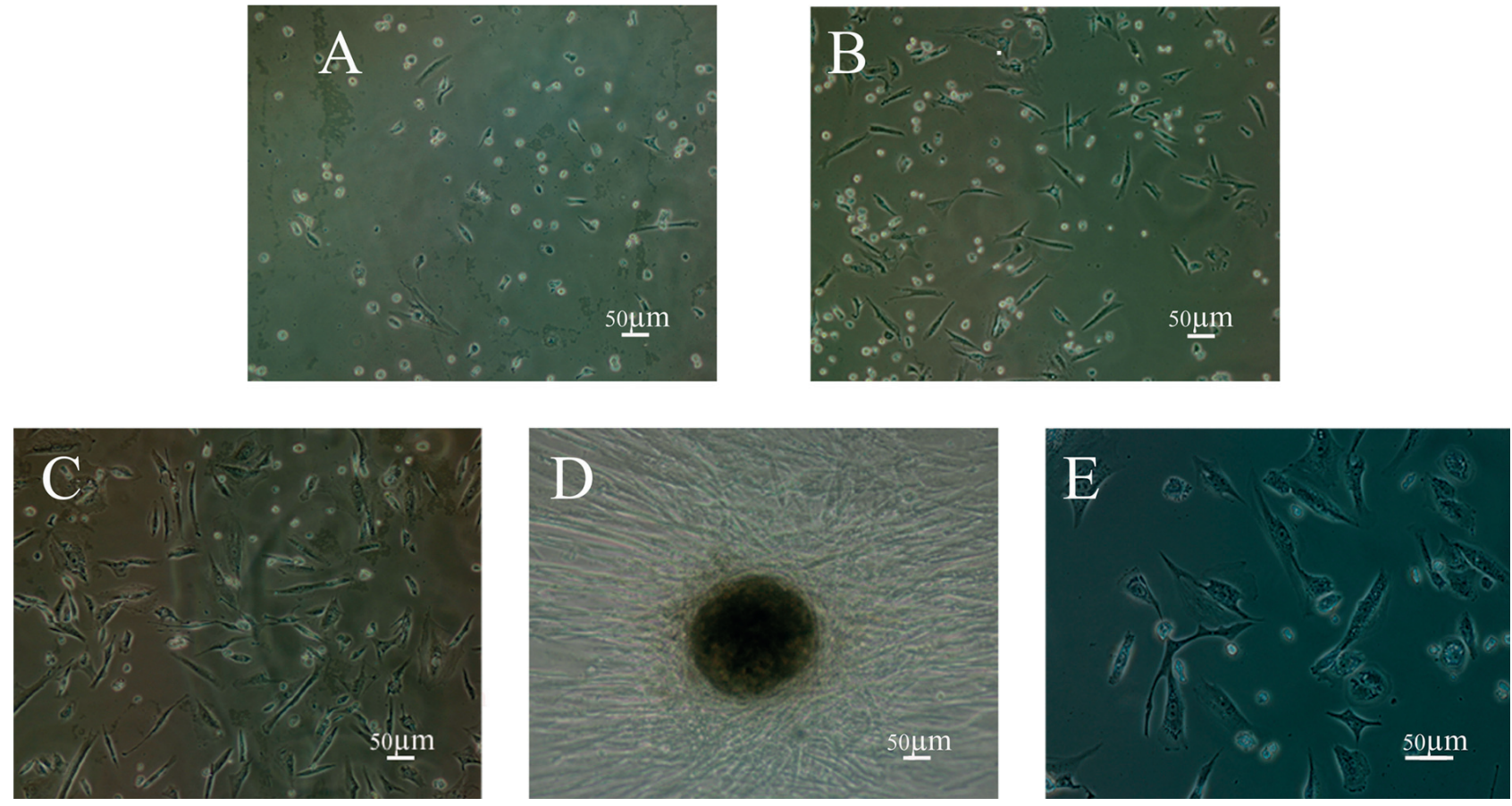

Fig. 2. Growth characterization of hAM-dMSCs in primary culture. At 3 passage in vitro, the shape of hAM-dMSCs grown into bipolar fibroblast-like cells at $1 \mathrm{~d}$ (A) and polygonal fibroblast-like cells at $2 \mathrm{~d}$ (B); they became relatively homogeneous in shape at $10 \mathrm{~d}(\mathrm{C}, \mathrm{E})$, growing cell colonies, which appeared after three passages in vitro (D). Scale bars in all figures represent $25 \mu \mathrm{m}$, except (E) represents $50 \mu \mathrm{m}(\mathrm{n}=8)$.

phological characteristics and surface antigens of hAMdMSCs were not significantly different between samples labeled with various concentrations of QDs and unlabeled controls, all of which were $>80 \%$ viable (Fig. 5, 6).

\section{DISCUSSION}

Stem cells play an important role in the regeneration of damaged tissues or the recovery of functional deficits in human disease. Mesenchymal stem cells (MSCs) have been found in multiple tissues, including bone marrow, adipose tissue, the umbilical cord and Wharton's jelly, amongst others ${ }^{12,13}$. To date, many studies both in vitro and in vivo have proved that MSCs can differentiate into epidermal-like cells or secrete specific epidermal cell proteins in specific chemical and cytokine microenvironments ${ }^{14-17}$. MSCs transplanted into animal models of damaged skin can survive, migrate, proliferate and differentiate, and have been shown to repair skin damage and improve the recovery of functional skin ${ }^{18-22}$.

The amniotic membrane displays many advantageous characteristics; it is easy to obtain at low cost, has little antigenic and anti-bacterial activity, and can be stored. Interestingly, hAM-dMSCs can be obtained from the amniotic membrane of discarded placental tissue, which provides a novel and attractive stem cell source and would avoid any ethical controversy. Many studies have demonstrated that special surface antigens, such as CD29, CD44, CD90 and CD105, can be detected on MSCs from different tissues ${ }^{23-25}$. In our study, MSCs isolated from human amniotic membrane showed high expression levels of CD29, CD44, CD90 and CD105, and were negative for CD31, CD34, CD45 and CD106; additionally, the morphological and growth characteristics of hAM-dMSCs were similar to MSCs from other tissues. If hAM-dMSCs are to be used to repair damaged skin, it is important to identify a highly effective method of labeling hAM-dMSCs before transplantation.

In our study we aimed to solve this problem, but monitoring transplanted cells in vivo is a challenge for the assessment of successful cell therapy. The identification of a noninvasive and nontoxic method is important for cell tracking in vivo. Recently, the use of fluorescent agents has begun to play an important role in the study of cellular structure, labeling of cellular molecules, and monitoring of different cells both in vivo and in vitro. However, traditional fluorescent agents have certain disadvantages, including broad emission spectra, limited brightness and low photostability, that limit long-term observation. Quantum dots (QDs) - small (1-10 nm) semiconductor nanocrystalloid particles with high durability and unique optical properties - are one of the most exciting materials in nanobiotechnology ${ }^{26,27}$. Compared with traditional fluorescent agents, quantum dots have nano-scale characteristics and bright and stable emission spectra. QDs have been used for labeling living cells and tissues. Endocytosis/phagocytosis or uptake by specific receptors or channels are processes used by many cell types to internalize larger proteins or nutrients. Recently, reports have indicated that QDs can be internalized into living cells through such endocytotic mechanisms. Once inside cells, QDs still retain their spectral profile. In our study, 

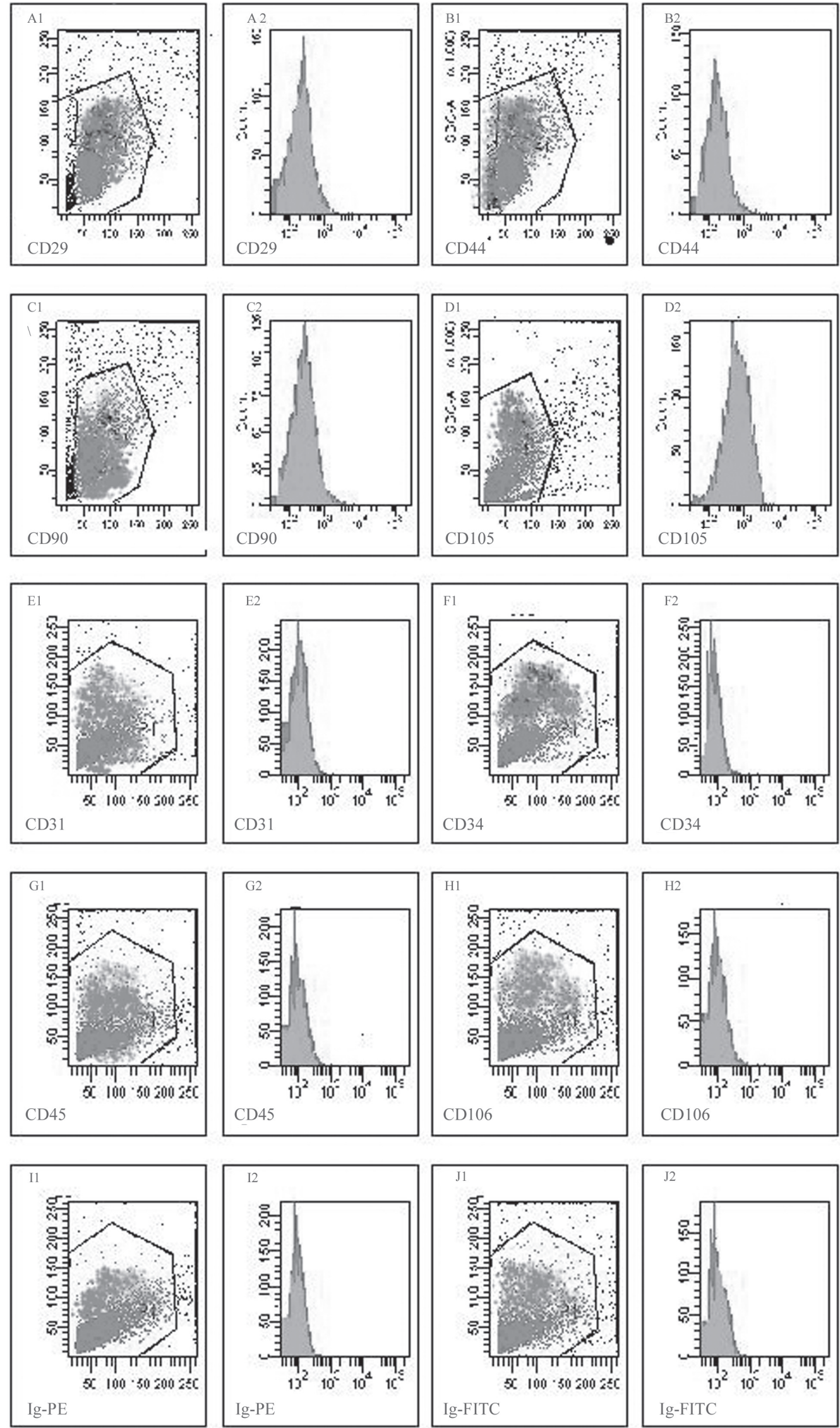

Fig. 3. Surface antigens of hAM-dMSCs in primary culture. In flow cytometry, hAM-dMSCs at passage 3 were positive for CD29 (A1, A2), CD44 (B1, B2), CD90 (C1, C2) and CD105 (D1, D2), and negative for CD31(E1, E2), CD34 (F1, F2), CD45 (G1, G2) and CD106 (H1, H2). Mouse IgG conjugated with FITC or PE served as the negative controls (I1, I2) and (J1, J2). Data are shown as the mean \pm standard error (S.E.) $(n=8)$. 
$100 \%$ of hAM-dMSCs were consistently loaded with different amount of QDs at different times. If they are to be used in vivo, these particles must be characterized to assess their biocompatibility. This is in comparison with other nanoparticles, such as iron nanoparticles, which have proven to be biocompatible. QDs as new materials have not been completely characterized for their toxicity in hAM-dMSCs.

Materials which affect the cell and allow viability of more than $80 \%$ are recognized as biocompatible ${ }^{28}$. In our experiments on QD-labeled hAM-dMSCs, results of the MTT assay revealed no significant differences between cells labeled with QDs at concentrations of $0.75 \mu \mathrm{g} / \mathrm{mL}$ and $1.5 \mu \mathrm{g} / \mathrm{mL}$ at $1 \mathrm{~d}$, with cell viability of all samples of $>80 \%$. After $1 \mathrm{~d}$, the viability of QD-labeled hAMdMSCs was $>80 \%$ when the initial QD concentration was $0.75 \mu \mathrm{g} / \mathrm{mL}$. High concentrations of QDs exhibited acute and chronic cytotoxic effects on hAM-dMSCs; the cell viability of samples was $<80 \%$ when the initial QD concentrations were $3.0 \mu \mathrm{g} / \mathrm{mL}$ at $1 \mathrm{~d}$ and $1.5 \mu \mathrm{g} / \mathrm{mL}$ at $2 \mathrm{~d}$. Our study revealed that concentrations $\leq 0.75 \mu \mathrm{g} / \mathrm{mL}$ were safe for QD labeling of hAM-dMSCs. hAM-dMSCs labeled with safe concentrations of QDs and harvested at 1, 2 and $4 \mathrm{~d}$ expressed CD29, CD44, CD90, CD105, CD31, CD34, CD45 and CD106, which were similar to unlabeled samples in primary culture (3 passages).

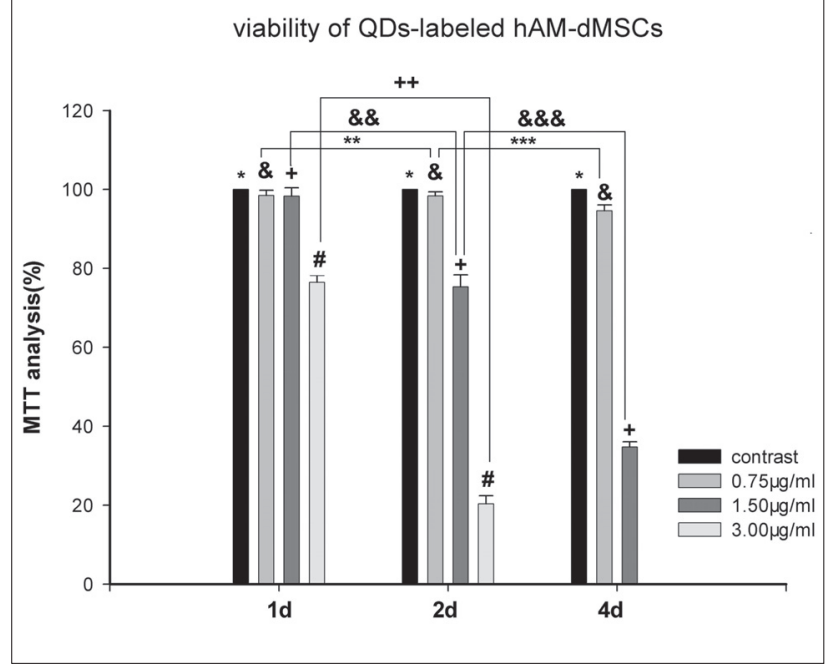

Fig. 4. QDs affect viability of hAM-dMSCs. In MTT assay, hAM-dMSCs were culturing in different conversation of QDs suspension under 1, 2 and $4 \mathrm{~d}$. The data are presented as the mean \pm standard error (S.E.). $1 \mathrm{~d},{ }^{*} P>0.05 \&, \& P>0.05 \#$, $\# P<0.05+; 2 \mathrm{~d}, * P>0.05 \&, \& P<0.05 \#, \# P<0.05+; 4 \mathrm{~d}$, * $P<0.05 \&, \& P<0.05 \#$; ** $P>0.05$; ** $P<0.05 ; P<0.05$; $P<0.05 ;++P<0.05(\mathrm{n}=8)$
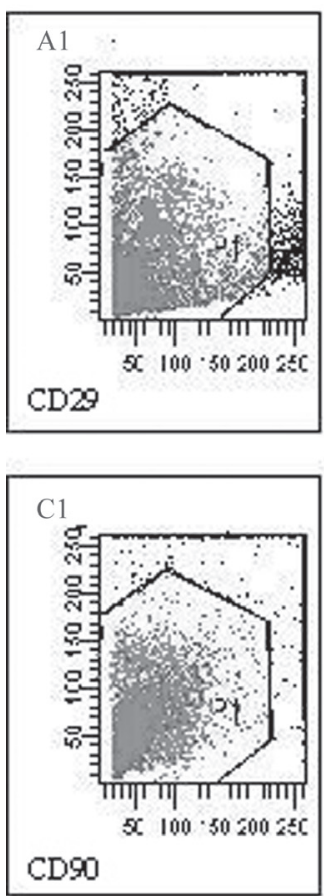
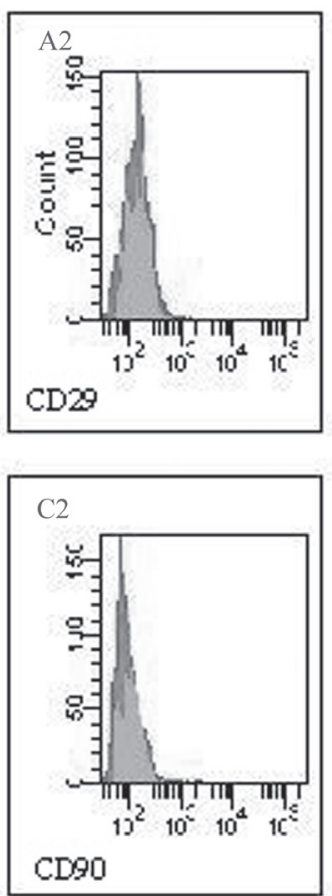
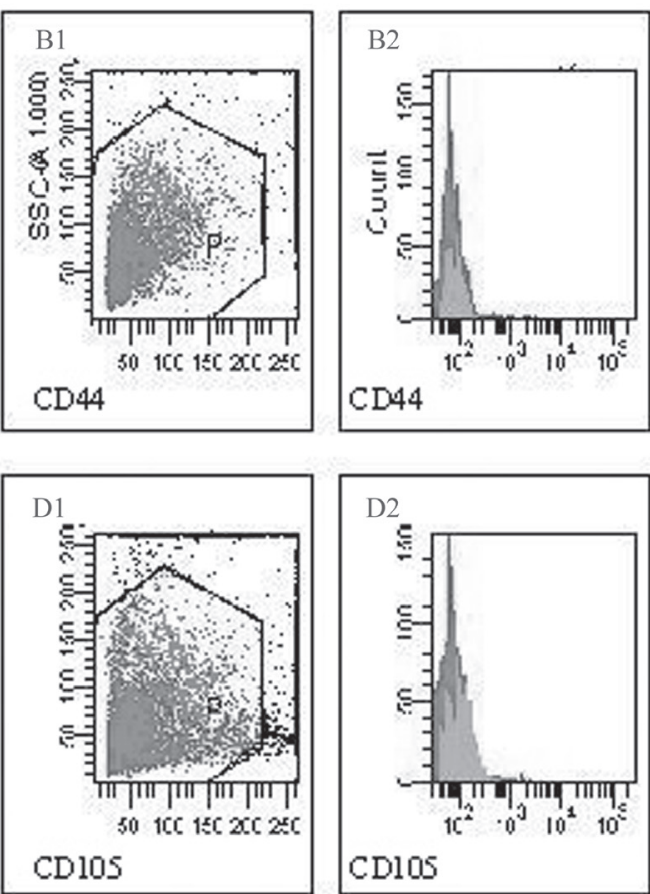

Fig. 5. Surface antigens of hAM-dMSCs were cultured in the QDs suspension. In flow cytometry, hAMdMSCs labeled with $0.75 \mu \mathrm{g} / \mathrm{mL}$ QDs were positive for CD29 (A1, A2), CD44 (B1, B2), CD90 (C1, C2) and CD105 (D1, D2) at 4 d. Data are shown as the mean \pm standard error (S.E.) $(n=8)$. 

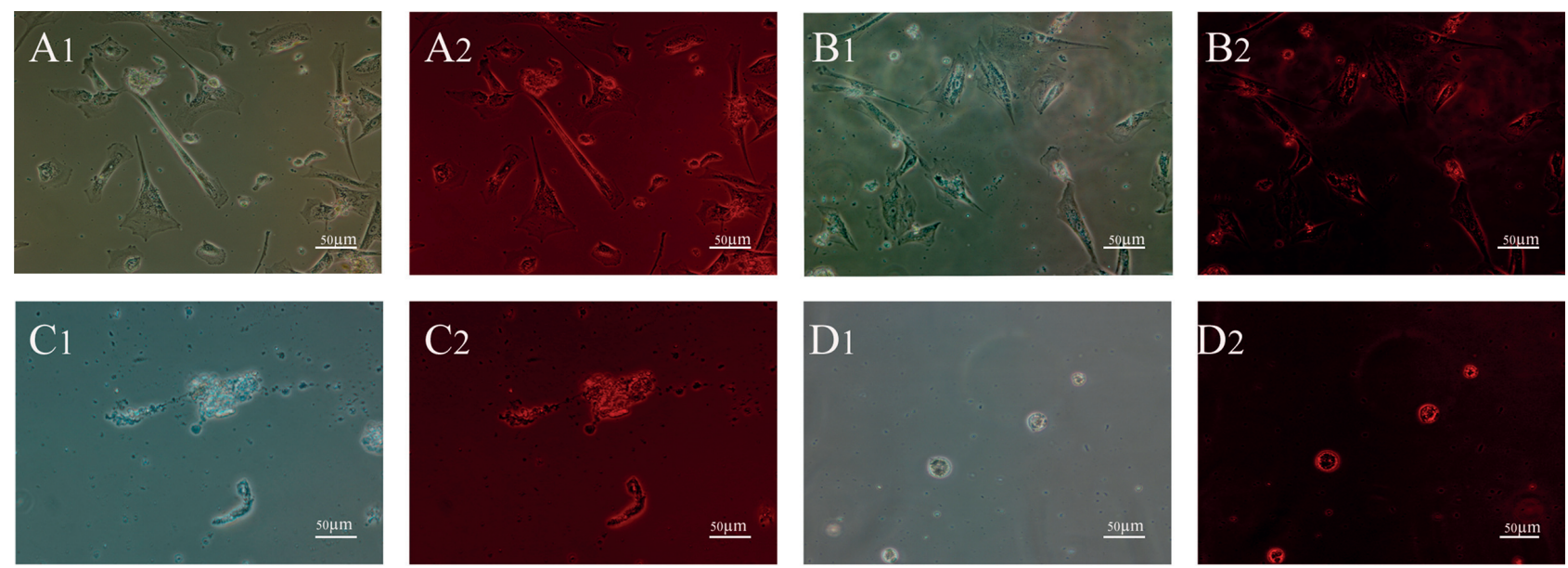

Fig. 6. Images of QD-labeled hAM-dMSCs. The images of QDs-labeled hAM-dMSCs before and after fluorescence microscope. Images of $0.75 \mu \mathrm{g} / \mathrm{mL}$ QD-labeled hAM-dMSCs which excite red color and keep normal morphological shape at $2 \mathrm{~d}$ (A1 before fluorescence microscope, A2 after fluorescence microscope) and 4d (B1 before fluorescence microscope, B2 after fluorescence microscope). Images of $3.0 \mu \mathrm{g} / \mathrm{mL}$ QD-labeled hAM-dMSCs which were taken off the surface of the cell culture dish and shaped spherocyte at 1d (A3 before fluorescence microscope, B3 after fluorescence microscope ). Images of $1.5 \mu \mathrm{g} / \mathrm{mLQD}$-labeled hAMdMSCs which excite red color and lost fibroblast-like morphological shape at 2d (A4 before fluorescence microscope, B4 after fluorescence microscope ) Scale bar $=25 \mu \mathrm{m}(\mathrm{n}=8)$.

\section{CONCLUSION}

We isolated hAM-dMSCs with high expression levels of CD29, CD44, CD90 and CD105. Safe concentrations of QDs for labeling of hAM-dMSCs, resulting in cell viabilities $>80 \%$ were $\leq 0.75 \mu \mathrm{g} / \mathrm{mL}$ from 1 to $4 \mathrm{~d}$. These concentrations do not alter the morphological characteristics of hAM-dMSCs or their expression of specific surface antigens such as CD29, CD44, CD90 and CD105.

\section{ACKNOWLEDGMENT}

We thank Shoumin Xi, M.D., Department of Pharmacology, Medical School of Henan University of Science and Technology, for the supply of laboratory.

Author contributions: GW: literature search; GZ: manuscript writing; CW: study design; HW: data collection; BY: data analysis; FG: data interpretation; DL: statistical analysis, figures; XF: final approval.

Conflict of interest statement: The authors state that there are no conflicts of interest regarding the publication of this article.

\section{REFERENCES}

1. Laco F, Kun M, Weber HJ, Ramakrishna S, Chan CK. The dose effect of human bone marrow-derived mesenchymal stem cells on epidermal development in organotypic co-culture. Dermatol Sci 2009;55:15060.

2. Ma K, Laco F, Ramakrishna S, Liao S, Chan CK. Differentiation of bone marrow-derived mesenchymal stem cells into multi-layered epidermis-like cells in 3D organotypic coculture. Biomaterials 2009;30:3251-8.

3. Agay D, Scherthan H, Forcheron F, Grenier N, Hérodin F, Meineke V Drouet M. Multipotent mesenchymal stem cell grafting to treat cu- taneous radiation syndrome: development of a new minipig model. Exp Hematol 2010;38:945-56.

4. Mendonça JJ, Juiz-Lopez P. Regenerative facial reconstruction of terminal stage osteoradionecrosis and other advanced craniofacial diseases with adult cultured stem and progenitor cells. Plast Reconstr Surg 2010;126:1699-1709.

5. Biju V, Itoh T, Ishikawa M. Delivering quantum dots to cells: bioconjugated quantum dots for targeted and nonspecific extracellular and intracellular imaging. Chem Soc Rev 2010;39:3031-56.

6. Hahn MA, Keng PC, Krauss TD. Flow cytometric analysis to detect pathogens in bacterial cell mixtures using semiconductor quantum dots. Anal Chem 2008;80:864-72.

7. Muro E, Pons T, Lequeux N, Fragola A, Sanson N, Lenkei Z, Dubertret B. Small and stable sulfobetaine zwitterionic quantum dots for functional live-cell imaging. Am Chem Soc 2010;132:4556-7.

8. Shiohara A, Prabakar S, Faramus A, Hsu CY, Lai PS, Northcote PT, Tilley RD. Sized controlled synthesis, purification, and cell studies with silicon quantum dots. Nanoscale 2010;3:3364-70.

9. Pinaud F, King D, Moore HP, Weiss S. Bioactivation and cell targeting of semiconductor CdSe/ZnS nanocrystals with phytochelatin-related peptides. Am Chem Soc 2004;126:6115-23.

10. Xie M, Liu HH, Chen P, Zhang ZL, Wang XH, Xie ZX, Du YM, Pan BQ, Pang DW. CdSe/ZnS-labeled carboxymethyl chitosan as a bioprobe forlive cell imaging. Chem Commun (Camb) 2005;44:5518-20.

11. Li GX, Yang B, Guan FX. Isolation, culture and identification of human amniotic-derived mesenchymal stem cells. Zhengzhou Daxue Xuebao 2006;412:244-6 (in Chinese).

12. Riekstina U, Cakstina I, Parfejevs V, Hoogduijn M, Jankovskis G, Muiznieks I, Muceniece R, Ancans J. Embryonic stem cell marker expression pattern in human mesenchymal stem cells derived from bone marrow, adipose tissue, heart and dermis. Stem Cell Rev 2009;5:378-86.

13. Hattori H, Sato M, Masuoka K, Ishihara M, Kikuchi T, Matsui T, Takase B, Ishizuka T, Kikuchi M, Fujikawa K, Ishihara M. Osteogenic potential of human adipose tissue-derived stromal cells as an alternative stem cell source.Cells Tissues Organs 2004;178:2-12.

14. Baer PC, Schubert R, Bereiter-Hahn J, Plösser M, Geiger H. Expression of a functional epidermal growth factor receptor on human adiposederived mesenchymal stem cells and its signaling mechanism. Eur J Cell Biol 2009;88:273-83.

15. Li L, Fukunaga-Kalabis M, Yu H, Xu X, Kong J, Lee JT, Herlyn M. Human dermal stem cells differentiate into functional epidermal melanocytes. J Cell Sci 2010;123:853-60.

16. Soulez M, Sirois I, Brassard N, Raymond MA, Nicodème F, Noiseux N, Durocher Y, Pshezhetsky AV, Hébert MJ. Epidermal growth factor 
and perlecan fragments produced by apoptotic endothelial cells co-ordinately activate ERK1/2-dependent antiapoptotic pathways in mesenchymal stem cells. Stem Cells 2010;28:810-20.

17. Zabierowski SE, Fukunaga-Kalabis M, Li L, Herlyn M. Dermis-derived stem cells: a source of epidermal melanocytes and melanoma? Pigment Cell Melanoma Res 2011;24:422-9.

18. Alexeev V, Uitto J, Igoucheva O. Gene expression signatures of mouse bone marrow-derived mesenchymal stem cells in the cutaneous environment and therapeutic implications for blistering skin disorder. Cytotherapy 2011;13:30-45.

19. Kim SS, Song CK, Shon SK, Lee KY, Kim CH, Lee MJ, Wang L. Effects of human amniotic membrane grafts combined with marrow mesenchymal stem cells on healing of full-thickness skin defects in rabbits. Cell Tissue Res 2009;336:59-66.

20. Rasulov MF, Vasilchenkov AV, Onishchenko NA, Krasheninnikov ME, Kravchenko VI, Gorshenin TL, Pidtsan RE, Potapov IV. First experience of the use bone marrow mesenchymal stem cells for the treatment of a patient with deep skin burns. Bull Exp Biol Med 2005;139:141-4.

21. Sasaki M, Abe R, Fujita Y, Ando S, Inokuma D, Shimizu H. Mesenchy mal stem cells are recruited into wounded skin and contribute to wound repair by transdifferentiation into multiple skin cell type. $J$ Immunol 2008;180:2581-7.
22. Yang M, Li Q, Sheng L, Li H, Weng R, Zan T. Bone marrow-derived mesenchymal stem cells transplantation accelerates tissue expansion by promoting skin regeneration during expansion. Ann Surg 2011;253:202-9.

23. Arufe MC, De la Fuente A, Fuentes I, de Toro FJ, Blanco FJ. Chondrogenic potential of subpopulations of cells expressing mesenchymal stem cell markers derived from human synovial membranes. J Cell Biochem 2010;111:834-45.

24. Kern S, Eichler H, Stoeve J, Klüter H, Bieback K. Comparative analysis of mesenchymal stem cells from bone marrow, umbilical cord blood, or adipose tissue. Stem Cells 2006;24: 1294-1301.

25. Pittenger MF, Mackay AM, Beck SC Jaiswal RK, Douglas R, Mosca JD, Moorman MA, Simonetti DW, Craig S, Marshak DR. Multilineage potential of adult human mesenchymal stem cells. Science 1999;284:143-7.

26. Archer PI, Santangelo SA, Gamelin DR. Inorganic cluster syntheses of TM2+-doped quantum dots (CdSe, CdS, CdSe/CdS): physical property dependence on dopant locale. J Am Chem Soc 207;129:9808-18.

27. Cao YC, Wang Z, Wang HQ, Wang JH, Hua XF, Jin X, Yang L, Huang ZL, Liu MX, Zhao YD. Enhanced optical property of Au coated polystyrene beads for multi-color quantum dots encoding. J Nanosci Nanotechnol 2009;9:1778-84.

28. Zborowski M, Chalmers JJ. Magnetic cell separation. Amsterdam, Elsevier, 2008. 